\title{
Vertebrobasilar artery junction aneurysm associated with fenestration
}

\author{
Javier Espinoza Bentura', Eberval G. Figueiredo², \\ Bernardo A. de Monaco ${ }^{3}$, Manoel J. Teixeira ${ }^{4}$
}

Fenestration of an intracranial artery is a rare occurrence. After the vertebral artery, the basilar artery is the second most frequent site of fenestration of intracranial arteries ${ }^{1,2}$. Alike arterial bifurcations, fenestrations resulting from developmental anomalies have a tendency to develop aneurysms ${ }^{3}$.

Fenestration of the proximal basilar artery aneurysms located at the vertebrobasilar junction may be associated with aneurysms ${ }^{1,4,5}$. Aneurysms arising from the posterior circulation are estimated to be less than $15 \%$ of all intracranial aneurysms. The morbidity and mortality of open surgery for posterior circulation aneurysms are higher than those of the anterior circulation $^{6}$. Endovascular treatment of vertebrobasilar junction aneurysms with Guglielmi Detachable Coils (GDC) can provide an alternative method of treatment $t^{1,2}$.

We present a fenestration of vertebral artery associated with intracranial aneurysm and discuss its pathogenesis and management.

\section{CASE}

A 45-years-old female complained of intense and sudden headache. At the emergency room she was awake and neurological examination revealed neck stiffness. CT scan displayed subarachnoid hemorrhage. Vertebral angiogram revealed a vertebral aneurysm arising from a fenestration at the vertebral junction (Fig 1). Patient underwent a far lateral approach. The vertebral artery was dissected, and the IX, $\mathrm{x}$ and XI were exposed at the jugular foramen. Aneurism was exposed (Fig 2) and clipped (Fig 3). Postoperative period was uneventful. Patient has given consent to publish this case.

\section{DISCUSSION}

The basilar artery is formed as a result of the regression of the trigeminal arteries. When the embryo is about $4 \mathrm{~mm}$ long, the bilateral longitudinal neural arteries are connected laterally at multiple locations with the primitive hindbrain plexus. At this stage, two fusion phenomena take place, one at the pontomesencephalic sulcus that is related to the caudal divisions of the internal carotid arteries (ICAs), while the other fusion process involves the longitudinal neural artery system and vertebrobasilar maturation. Caudal basilar fusion occurs with late trigeminal involution and cranial basilar fusion with early involution, because the trigeminal artery is responsible for the flow changes in the verte-

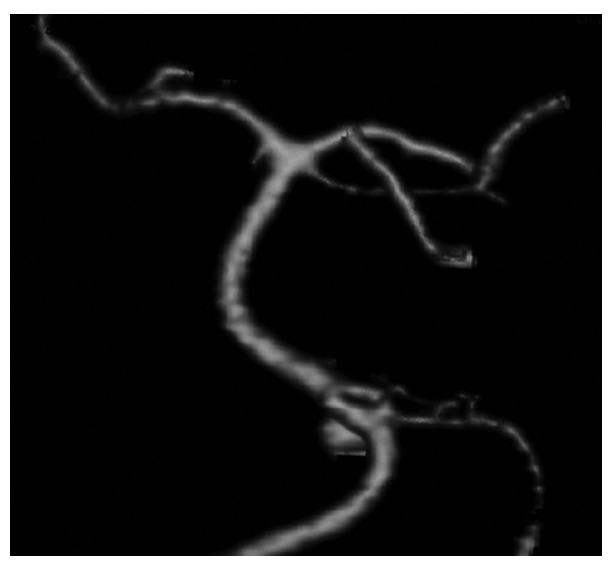

Fig 1. Vertebral angiogram depicts a vertebral junction aneurysm associated with fenestration. Frontal view.
Eberval G. Figueired

Rua Enéas de Carvalho Aguiar 255 05403-000 São Paulo SP - Brasil

E-mail: ebgadelha@yahoo.com

\section{ANEURISMA DA JUNÇÃO DA ARTÉRIA VERTEBROBASILAR ASSOCIADO A FENESTRAÇÃO}

${ }^{1} \mathrm{MD}$, Resident, Honorio Delgado Hospital Universidad Nacional de San Augustin, Orequipa, Peru; ${ }^{2} \mathrm{MD}, \mathrm{PHD}$, Supervisor, Head of Cerebrovacular Surgery, Division of Neurological Surgery University of São Paulo School of Medicine, São Paulo SP, Brazil; ${ }^{3} \mathrm{MD}$, Resident, Division of Neurological Surgery University of São Paulo School of Medicine, São Paulo SP, Brazil; ${ }^{4} \mathrm{MD}, \mathrm{PHD}$, Chairman and Director, Division of Neurological Surgery University of São Paulo School of Medicine, São Paulo SP, Brazil. 


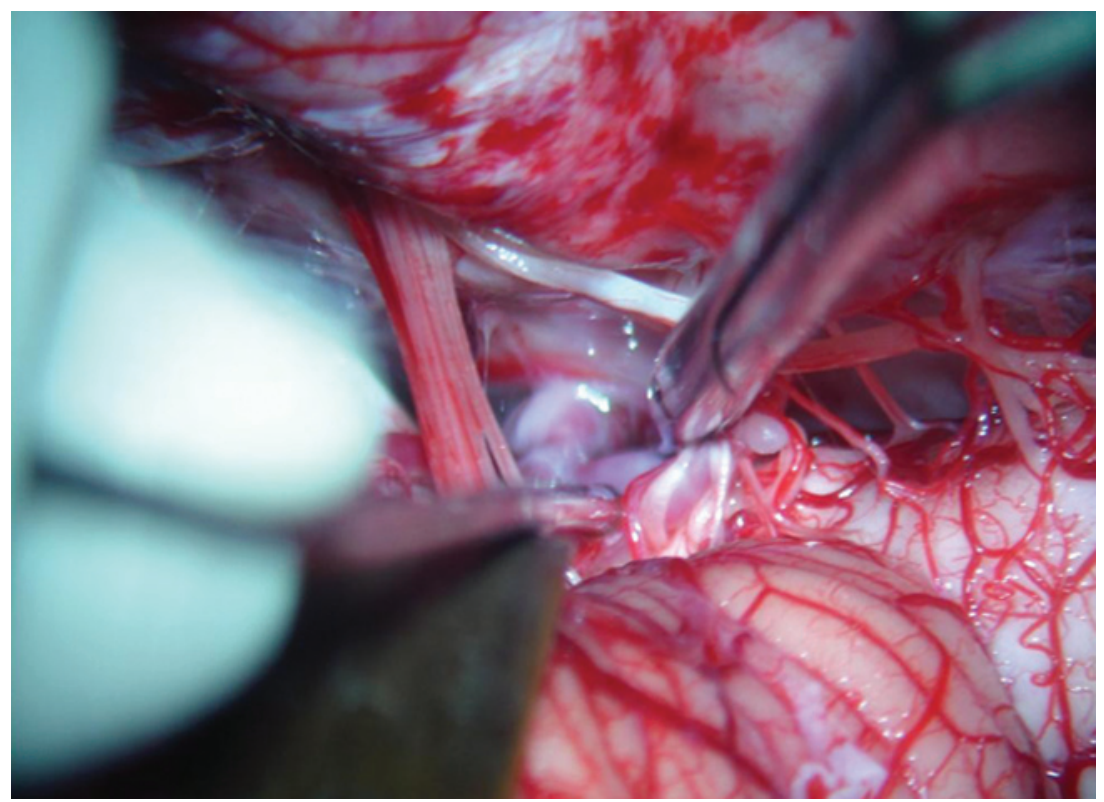

Fig 2. Microsurgical view. IX, X and XI nerves have been exposed and the vertebral junction has been visualized. Aneurysm has been identified and dissected.

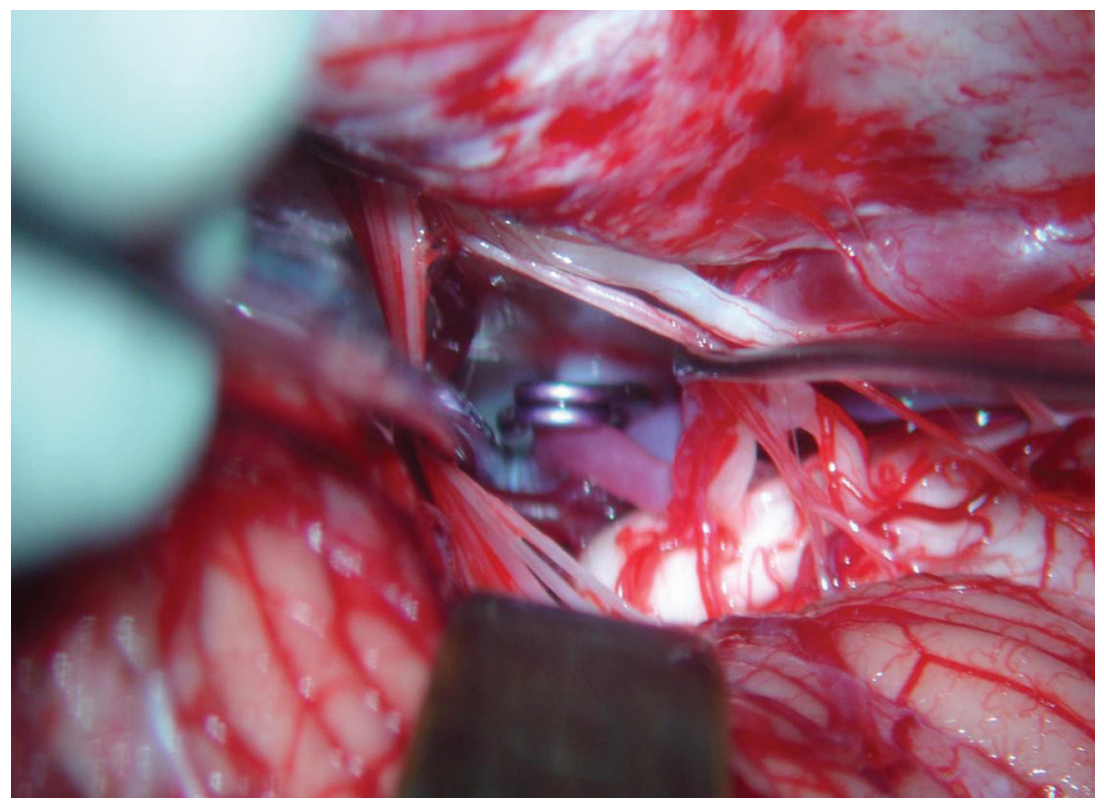

Fig 3. Aneurysm has been uneventfully clipped.

bral system during arterial development. The fusion process is typically completed when the embryo is $9 \mathrm{~mm}$ long (i.e. around the $5^{\text {th }}$ fetal week). Failure of fusion of the neural arteries with regression of the bridging vessels between longitudinal arteries is an explanation for the phenomenon of basilar artery fenestration.

A fenestration is defined as a single artery with two luminal channels. This is typically encountered in the vertebral artery or the ICAs in the neck. A lack of fusion of embryologically paired vessels, leads to segmentally un- fused arteries. This condition can only exist where two embryological arteries fuse during development. Therefore, the basilar artery or the anterior spinal artery can harbor unfused segments.

Duplication can occur where the "double lumen" is due to two embryologically different vessels that fuse during development; an additional vessel persists, whereas in fenestrations the two lumina correspond to a single artery. Non-fusion of the basilar artery usually involves the lower half of the vessel because of incomplete fusion of the lon- 
gitudinal arteries in the craniocaudal direction, although distal non-fusions have been reported and are very rare ${ }^{2,7}$.

Since the basilar artery develops by the midline fusion of the paired ventral longitudinal arteries, multiple variations of the vessel will occur depending on the fusion process of the longitudinal arteries and contribution of the segmental arteries.

Although rare, arterial fenestrations and unfused arterial segments can be associated with intracranial saccular aneurysms. The lateral walls of the fenestrated artery have a normal intrinsic architecture; however, the medial walls may divide the vascular channel into two distinct channels. The medial walls of the fenestration have focal defects at both ends. The media are absent locally with discontinuity of elastin at the proximal end of the fenestration. The subendothelium is thickened distally and thinned proximally. These structural changes at the proximal end of the fenestration are similar to those seen at cerebral artery bifurcations. The changes in the subendothelial structures are consistent with those produced by hemodynamic stress ${ }^{1,5,7-9}$.

The true frequency of fenestration of the basilar artery is difficult to ascertain, and the data vary according to type of series. The incidence of fenestration of the basilar artery is reported to be $1.3 \%$ to $6 \%$ in autopsy series and $0.02 \%$ to $0.6 \%$ in angiographic series. The discrepancy between the autopsy incidence and angiographic incidence of basilar artery fenestrations can be explained by the fact that in some fenestrations the divider is very thin and in most projections is angiographically occult ${ }^{1,2}$. The new radiological techniques such as $\mathrm{CT}$ angiography, $\mathrm{MR}$ angiography, and especially $3 \mathrm{D}$ digital subtraction angiography can be helpful in accurate pretreatment diagnosis ${ }^{2}$.
When a vertebrobasilar junction aneurysm is present, an associated fenestration should be strongly suspected ${ }^{5}$.

As with other intracranial aneurysms not associated with fenestration, basilar artery fenestration aneurysms can also be treated endovascularly with GDC. The typical origin of the vertebrobasilar junction aneurysm at the proximal portion of the fenestration, the complex geometry of the fenestration, the proximity of the lower cranial nerves, multiple small perforating arteries to the brain stem, and the difficulty of obtaining adequate surgical exposure have made the surgical treatment of these aneurysms difficult ${ }^{1,3,4}$. However the complex hemodynamics associated with these lesions may favor the surgical clipping as it provides well- known long term outcomes.

\section{REFERENCES}

1. Albanese $E$, Russo A, Ulm AJ. Fenestrated vertebrobasilar junction aneurysm: diagnostic and therapeutic considerations. J Neurosurg 2009;110:525-529.

2. Crivelli G, Bianchi M, Dario A, Dorizzi A. Saccular aneurysm associated with proximal basilar artery fenestration: case report. J Neurosurg Sci 1993;37:29-34.

3. Campos J, Fox AJ, Viñuela F, et al. Saccular aneurysms in basilar artery fenestration. AJNR Am J Neuroradiol 1987:8:233-236.

4. De Caro R, Serafini MT, Galli S, Parenti A, Guidolin D, Munari PF. Anatomy of segmental duplication in the human basilar artery. Possible site of aneurysm formation. Clin Neuropathol 1995;14:303-309.

5. Eustacchio S, Klein GE, Pendl G. Ruptured vertebrobasilar junction aneurysm associated with basilar artery fenestration. Acta Neurochir (Wien) 1997; 139:923-927.

6. Finlay HM, Canham PB. The layered fabric of cerebral artery fenestrations. Stroke 1994;25:1799-1806

7. Fujimoto K, Kawai S, Yonezawa T, et al, Basilar trunk aneurysms with associated fenestration treated by using Guglielmi Detachable Coils: two cases report. J Stroke Cerebrovasc Dis 2007:16:84-87.

8. Greenberg E, Katz J, Janardhan V, Riina H, Gobin P, Treatment of a giant vertebrobasilar artery aneurysm using stent grafts: case report. J Neurosurg 2007: 107:165-168.

9. Graves V, Strother C, Weir B, Duff T. Vertebrobasilar junction aneurysms associated with fenestration: treatment with Guglielmi Detachable Coils. AJNR 1996;17:35-40. 